TRANSACTIONS OF THE

AMERICAN MATHEMATICAL SOCIETY

Volume 362, Number 5, May 2010, Pages 2507-2523

S 0002-9947(09)04944-7

Article electronically published on December 2, 2009

\title{
ON ASYMPTOTIC TEICHMÜLLER SPACE
}

\author{
ALASTAIR FLETCHER
}

\begin{abstract}
In this article we prove that for any hyperbolic Riemann surface $M$ of infinite analytic type, the little Bers space $Q_{0}(M)$ is isomorphic to $c_{0}$. As a consequence of this result, if $M$ is such a Riemann surface, then its asymptotic Teichmüller space $A T(M)$ is bi-Lipschitz equivalent to a bounded open subset of the Banach space $l^{\infty} / c_{0}$. Further, if $M$ and $N$ are two such Riemann surfaces, their asymptotic Teichmüller spaces, $A T(M)$ and $A T(N)$, are locally bi-Lipschitz equivalent.
\end{abstract}

\section{INTRODUCTION}

1.1. Background. In this paper, we discuss an isomorphism theorem for certain Banach spaces of holomorphic quadratic differentials and applications to the biLipschitz class of asymptotic Teichmüller space.

The asymptotic Teichmüller space $A T(M)$ of a hyperbolic Riemann surface $M$ was introduced by Gardiner and Sullivan (cf. [9]) for the upper half-plane, and by Earle, Gardiner and Lakic for arbitrary hyperbolic Riemann surfaces (cf. [2, 3, 8]). Two quasiconformal mappings $f$ and $g$ on $M$ are called equivalent if there is an asymptotically conformal mapping $h$ of $f(M)$ onto $g(M)$ such that $g^{-1} \circ h \circ f$ is homotopic to the identity relative to the ideal boundary of $M$. Asymptotic Teichmüller space $A T(M)$ is the set of equivalence classes of quasiconformal mappings of $M$ under this relation. This definition is a variation of the definition of Teichmüller space $T(M)$ (see the books [6, 8, 10, 12, 20] and the references cited there for the extensive body of work on Teichmüller theory), where the mapping $h$ is required to be conformal.

Since conformal mappings are asymptotically conformal, there is a well-defined projection $\mathcal{P}: T(M) \rightarrow A T(M)$. If $M$ is a Riemann surface of finite analytic type, that is, a closed surface of finite genus with a finite number of points removed, then $A T(M)$ consists of one point.

Earle, Gardiner and Lakic further proved that $A T(M)$ has a complex manifold structure so that the quotient map $\mathcal{P}: T(M) \rightarrow A T(M)$ is holomorphic and further that the Bers embedding of $T(M)$ into $Q\left(M^{*}\right)$, where $M^{*}$ is the conjugate surface to $M$ and $Q(M)$ is the Banach space of holomorphic quadratic differentials on $M$ with bounded Bers norm, induces a locally biholomorphic map of $A T(M)$ into the Banach space $\widehat{Q}\left(M^{*}\right):=Q\left(M^{*}\right) / Q_{0}\left(M^{*}\right)$, where $Q_{0}(M) \subseteq Q(M)$ are those holomorphic quadratic differentials which vanish at infinity. This map is called

Received by the editors February 29, 2008.

2010 Mathematics Subject Classification. Primary 30F60.

Key words and phrases. Asymptotic Teichmüller space, little Bers space.

The author was supported by EPSRC grant EP/D065321/1.

(C)2009 American Mathematical Society Reverts to public domain 28 years from publication 2507 
the asymptotic Bers map. Earle, Markovic and Saric showed (cf. [4) that the asymptotic Bers map is a biholomorphic map from $A T(M)$ onto a bounded open set in $\widehat{Q}\left(M^{*}\right)$. For further recent work on asymptotic Teichmüller spaces and the asymptotic Bers map, see [18, 19, 25].

1.2. Motivation. The aim of this paper is to study the Banach space $\widehat{Q}(M)$ and the resulting implications for asymptotic Teichmüller space via the asymptotic Bers embedding. The following theorem was proved in [5].

Theorem A (Fletcher, 2006). Let $M$ and $N$ be Riemann surfaces of infinite analytic type (i.e., the unit disk or a Riemann surface of infinite genus or with an infinite number of punctures). Then the Bergman space $A^{1}(M)$ of $L^{1}$-integrable holomorphic quadratic differentials on $M$ is isomorphic to the sequence space $l^{1}$. Further, $A^{1}(M)$ is isomorphic to $A^{1}(N)$.

Examples of Riemann surfaces include the plane punctured at the Gaussian integer lattice points and an infinite genus surface. In particular, $M$ and $N$ are not assumed to be homeomorphic. Theorem A should be compared with the following result, which was used in [16] to generalise Royden's theorem on classifying biholomorphic maps between Teichmüller spaces for Riemann surfaces of infinite analytic type.

Theorem B (Markovic, 2003). Let $M$ and $N$ be two Riemann surfaces of infinite analytic type. If there exists a surjective linear isometry between $A^{1}(M)$ and $A^{1}(N)$, then the surfaces $M$ and $N$ are conformally equivalent.

Therefore, while we always have an isomorphism between $A^{1}(M)$ and $A^{1}(N)$ when $M$ and $N$ are of infinite type, if we have an isometry, then the surfaces must be conformally related. As a corollary to Theorem A, we have the following result, also proved in [5].

Theorem C (Fletcher, 2006). If $M$ and $N$ are Riemann surfaces of infinite analytic type, then $Q(M)$ is isomorphic to $l^{\infty}$ and $Q(M)$ is isomorphic to $Q(N)$. Further, there is a bi-Lipschitz map of $T(M)$ onto a bounded open subset of $l^{\infty}$. Given $[\mu]_{T(M)} \in T(M)$ and $[\nu]_{T(N)} \in T(N)$, there exist neighbourhoods $U_{1}, U_{2}$ of $[\mu]_{T(M)}$ and $[\nu]_{T(N)}$, respectively, and a bi-Lipschitz map between $U_{1}$ and $U_{2}$. That is, $T(M)$ and $T(N)$ are locally bi-Lipschitz equivalent.

The aim of this paper is to find analogous results to Theorems $\mathrm{A}$ and $\mathrm{C}$ for asymptotic Teichmüller space. As is well known, the Banach dual of $Q_{0}(M)$ can be identified with $A^{1}(M)$ (cf. [17, 22, 23]) and the Banach dual of $c_{0} \subset l^{\infty}$, the space of bounded sequences which converge to zero, can be identified with $l^{1}$. However, the author is not aware of any simple argument which would allow the isomorphism between $A^{1}(M)$ and $l^{1}$ in Theorem A to give an isomorphism between the pre-duals $Q_{0}(M)$ and $c_{0}$. One aim of this paper is to construct such an isomorphism using a variation on the argument used to prove Theorem A in [5].

\subsection{Results.}

Theorem 1.1. Let $M$ and $N$ be Riemann surfaces of infinite analytic type. Then there exists an isomorphism $\beta_{M}: Q_{0}(M) \rightarrow c_{0}$. Further, $Q_{0}(M)$ and $Q_{0}(N)$ are isomorphic. 
As with Theorem A, the surfaces $M$ and $N$ are not assumed to be homeomorphic in Theorem 1.1. Note that Theorem 1.1 implies Theorem A by considering the Banach duals of $Q_{0}(M)$ and $c_{0}$. The conclusion of this theorem is that there exists a constant $C_{M}$ depending only on $M$ such that

$$
\frac{\left\|\beta_{M}(\varphi)\right\|_{l^{\infty}}}{C_{M}} \leq\|\varphi\|_{Q} \leq C_{M}\left\|\beta_{M}(\varphi)\right\|_{l^{\infty}}
$$

for all $\varphi \in Q_{0}$, where $\|.\|_{Q}$ denotes the Bers norm on $Q(M)$. Whether one can find some universal constant $C$ in place of $C_{M}$ in (1.1) which holds over all Riemann surfaces $M$ is an interesting question.

Corollary 1.2. Let $M$ and $N$ be two Riemann surfaces of infinite analytic type. Then there exists an isomorphism $\widehat{\beta_{M}}: \widehat{Q}(M) \rightarrow l^{\infty} / c_{0}$. Further, $\widehat{Q}(M)$ is isomorphic to $\widehat{Q}(N)$.

By combining the asymptotic Bers embedding with $\widehat{\beta_{M}}$, we have the following result.

Theorem 1.3. If $M$ is a Riemann surface of infinite analytic type, then there exists a bi-Lipschitz mapping of asymptotic Teichmüller space $A T(M)$ onto a bounded open subset of $l^{\infty} / c_{0}$.

Remark 1.1. By results in [4], $A T(M)$ is contractible. Corollary 1.3 then implies that the bi-Lipschitz image of $A T(M)$ in $l^{\infty} / c_{0}$ is also contractible.

Using the complex manifold structure of asymptotic Teichmüller space gives the following.

Theorem 1.4. Let $M$ and $N$ be Riemann surfaces of infinite analytic type. Given $[\mu]_{A T(M)} \in A T(M)$ and $[\nu]_{A T(N)} \in A T(N)$, there exist neighbourhoods $U_{1}, U_{2}$ of $[\mu]_{A T(M)}$ and $[\nu]_{A T(N)}$, respectively, and a bi-Lipschitz map between $U_{1}$ and $U_{2}$. That is, $A T(M)$ and $A T(N)$ are locally bi-Lipschitz equivalent.

The space $T_{0}(M)$ is defined to be the subset of $T(M)$ with asymptotically conformal representatives. Recalling the projection $\mathcal{P}: T(M) \rightarrow A T(M), T_{0}(M)$ is $\mathcal{P}^{-1}\left([0]_{A T(M)}\right)$. It is proved in [3, 8] that $T_{0}(M)$ is a closed complex submanifold of $T(M)$.

Corollary 1.5. The set $T_{0}(M)$ is homeomorphic to a bounded subset of $c_{0}$.

The paper is organised as follows. In $§ 2$, we recall the Bers and Bergman spaces of holomorphic quadratic differentials, define Teichmüller space and asymptotic Teichmüller space, recall Beltrami differentials, Schwarzian derivatives and the Bers embedding and asymptotic Bers embedding. In $\S 3$, we prove Theorem 1.1. The proof uses the theory of Bergman kernels, and the relevant results are recalled or proved therein. In $\S 4$, we prove the consequences of Theorem 1.1 stated above.

\section{Notation}

2.1. Banach spaces of holomorphic quadratic differentials. Let

$$
\mathbb{D}=\{z \in \mathbb{C}:|z|<1\}
$$


be the unit disk. The Bergman space $A^{1}(\mathbb{D})$ is the Banach space of holomorphic quadratic differentials $f=f d z^{2}$ on $\mathbb{D}$ with finite norm

$$
\|f\|_{1}=\int_{\mathbb{D}}|f(z)| d \sigma(z)<\infty
$$

for $f \in A^{1}(\mathbb{D})$, where $\sigma$ is two-dimensional area measure. The Bers space $Q(\mathbb{D})$ is the Banach space of holomorphic quadratic differentials on $\mathbb{D}$ with finite hyperbolicsupremum norm given by

$$
\|\varphi\|_{Q}=\sup _{z \in \mathbb{D}} \rho^{-2}(z)|\varphi(z)|<\infty
$$

for $\varphi \in Q(\mathbb{D})$, where

$$
\rho(z)=2 /(1-|z|)^{2}
$$

is the hyperbolic density on $\mathbb{D}$. The little Bers space $Q_{0}(\mathbb{D})$ is the subset of $Q(\mathbb{D})$ for which

$$
\limsup _{|z| \rightarrow 1} \rho^{-2}(z)|\varphi(z)|=0 .
$$

In [13, Lindenstrauss and Pelczynski proved that $A^{1}(\mathbb{D})$ is isomorphic to the sequence space $l^{1}$ by using a result of Shields and Williams [23] that there is a projection from $L^{1}(\mathbb{D})$ onto $A^{1}(\mathbb{D})$. Further, Wojtaszczyk $[24$ exhibited an explicit basis for $A^{1}(\mathbb{D})$ by using spline systems. The little Bers space is known to be isomorphic to $c_{0}$ and by using projections given in 23], other weighted spaces of holomorphic functions can be shown to be isomorphic to $c_{0}$ (see [14, 15]).

A Riemann surface $M$ is hyperbolic if its universal covering surface is conformally equivalent to the unit disk $\mathbb{D}$. Such a Riemann surface carries a hyperbolic metric with density $\rho_{M}$, which is the pullback of the hyperbolic density on $\mathbb{D}$ given in (2.1) via the projection $\pi: \mathbb{D} \rightarrow M$. Let $G$ be the group of covering transformations and let $\Omega$ be a fundamental region for $M$. Define $Q(\mathbb{D}, G)$ to be the subset of $Q(\mathbb{D})$ consisting of those $\varphi \in Q(\mathbb{D})$ which satisfy the $G$-equivariance

$$
(\varphi \circ g)\left(g^{\prime}\right)^{2}=\varphi
$$

for all $g \in G$. The relation (2.2) implies that a quadratic differential $\varphi \in Q(\mathbb{D}, G)$ projects to a quadratic differential $\widetilde{\varphi}$ on $M$ which satisfies

$$
\|\widetilde{\varphi}\|_{Q(M)}=\sup _{p \in M} \rho_{M}^{-2}(p)|\widetilde{\varphi}(p)|<\infty .
$$

Conversely, a quadratic differential $\widetilde{\varphi}$ on $M$ lifts to a quadratic differential $\varphi$ on $\Omega$ and we use the relation (2.2) to extend the domain of $\varphi$ to $\mathbb{D}$. We can therefore identify the Bers space $Q(M)$, consisting of holomorphic quadratic differentials $\varphi$ on $M$ for which $\|\varphi\|_{Q}<\infty$, with $Q(\mathbb{D}, G)$. For brevity, we write $\|.\|_{Q}$ instead of $\|\cdot\|_{Q(M)}$ since it will be clear which Bers space is under consideration. The Bergman space $A^{1}(M)$ is the Banach space of holomorphic quadratic differentials $\widetilde{\varphi}$ on $M$ for which

$$
\|\widetilde{\varphi}\|_{1}=\int_{M}|\widetilde{\varphi}| d \sigma_{M}<\infty
$$

where $\sigma_{M}$ is 2-dimensional area measure on $M$.

A holomorphic quadratic differential $\widetilde{\varphi} \in Q(M)$ vanishes at infinity if for all $\epsilon>0$, there exists a compact set $E \subset M$ such that

$$
\sup _{p \in M \backslash E} \rho_{M}^{-2}(p)|\widetilde{\varphi}(p)|<\epsilon .
$$


The little Bers space $Q_{0}(M) \subset Q(M)$ is the subspace consisting of those holomorphic quadratic differentials which vanish at infinity. As is well known, the Banach dual of $Q_{0}(M)$ can be identified with $A^{1}(M)$ and the double dual of $Q_{0}(M)$ can be identified with $Q(M)$ (see for example [17, 22, 23]). We will write $\widehat{Q}(M)$ for the quotient Banach space $Q(M) / Q_{0}(M)$.

2.2. Teichmüller space and asymptotic Teichmüller space. Let $\pi: \mathbb{D} \rightarrow M$ be a holomorphic universal covering of the hyperbolic Riemann surface $M$. Every quasiconformal self-mapping $f$ of $M$ lifts to a quasiconformal self-mapping $\tilde{f}$ of $\mathbb{D}$. The map $f$ is called Teichmüller trivial if it has a lift $\widetilde{f}$ whose continuous extension to $\overline{\mathbb{D}}$ is the identity on $\partial \mathbb{D}$. The Teichmüller trivial quasiconformal self-mappings of $M$ form a group under composition. A quasiconformal map $f: M \rightarrow N$ between two Riemann surfaces is called asymptotically conformal if for every $\epsilon>0$, there exists a compact set $E \subset M$ such that $\left.f\right|_{E}$ is $(1+\epsilon)$-quasiconformal.

The Teichmüller space $T(M)$ is defined as a quotient space of the set of all quasiconformal maps with domain $M$ in the following way. The quasiconformal maps $f_{1}: M \rightarrow N_{1}$ and $f_{2}: M \rightarrow N_{2}$ are called Teichmüller equivalent if there exists a Teichmüller trivial map $h$ such that the mapping $f_{2} \circ h \circ f_{1}^{-1}$ from $N_{1}$ onto $\mathrm{N}_{2}$ is conformal. This defines an equivalence relation and $T(M)$ is defined to be the set of Teichmüller equivalence classes. Teichmüller space is equipped with a base-point, the Teichmüller equivalence class of the identity self-mapping of $M$.

The asymptotic Teichmüller space $A T(M)$ is defined as the quotient space of the same set of quasiconformal mappings with respect to the following equivalence relation. The quasiconformal maps $f_{1}: M \rightarrow N_{1}$ and $f_{2}: M \rightarrow N_{2}$ are called asymptotically equivalent if there exists a Teichmüller trivial map $h$ such that the mapping $f_{2} \circ h \circ f_{1}^{-1}$ from $N_{1}$ onto $N_{2}$ is asymptotically conformal. The set of asymptotic equivalence classes is the asymptotic Teichmüller space $A T(M)$ and the base-point is the asymptotic equivalence class of the identity self-mapping of $M$.

Since conformal maps are asymptotically conformal, there is a well-defined quotient map $\mathcal{P}: T(M) \rightarrow A T(M)$ that maps base-point to base-point. A class $[f]_{T(M)} \in T(M)$ is called asymptotically conformal if $\mathcal{P}\left([f]_{T(M)}\right)$ is the base-point of $A T(M)$. This happens if and only if $[f]_{T(M)}$ contains an asymptotically conformal representative. The set of asymptotically conformal classes in $T(M)$ is denoted by $T_{0}(M)$, and it is a closed submanifold of $T(M)$ (see [3, 8]).

If $M$ is a Riemann surface of finite analytic type, then $A T(M)$ consists of just one point (see [3]). In fact, in this case any quasiconformal mapping on $M$ has a quasiconformal mapping which is conformal outside a compact set in $M$ in its asymptotic Teichmüller class (see [18]).

2.3. Beltrami differentials. Recall $\pi: \mathbb{D} \rightarrow M$ is a holomorphic universal covering of the hyperbolic Riemann surface $M$. Let $G$ be the group of covering transformations for $\pi$. The set $L^{\infty}(\mathbb{D}, G)$ is the closed subspace of $L^{\infty}(\mathbb{D})$ consisting of those $\mu \in L^{\infty}(\mathbb{D})$ which satisfy the $G$-invariance condition

$$
(\mu \circ g) \frac{\overline{g^{\prime}}}{g^{\prime}}=\mu
$$

for all $g \in G$. 
The Beltrami differentials are those elements in the open unit ball $B(\mathbb{D}, G)$ of $L^{\infty}(\mathbb{D}, G)$ and $(2.3)$ implies that they project to Beltrami differentials, which are $(-1,1)$ differential forms, on $M$. This gives correspondences between $L^{\infty}(M)$, $B(M)$ and $L^{\infty}(\mathbb{D}, G), B(\mathbb{D}, G)$, respectively.

For each $\mu \in B(\mathbb{D}, G)$, let $f^{\mu}$ be the quasiconformal self-mapping of $\widehat{\mathbb{C}}=\mathbb{C} \cup\{\infty\}$ that maps $\mathbb{D}$ to itself, fixes $1,-1, i$, has the Beltrami differential $\mu$ in $\mathbb{D}$ and further satisfies $f^{\mu}\left(z^{*}\right)=\left(f^{\mu}(z)\right)^{*}$ for all $z \in \widehat{\mathbb{C}}$, where $z^{*}=1 / \bar{z}$ is reflection in $\partial \mathbb{D}$. Then (2.3) implies that

$$
g^{\mu}=f^{\mu} \circ g \circ\left(f^{\mu}\right)^{-1}
$$

is a Möbius transformation for all $g \in G$. The group $G^{\mu}=\left\{g^{\mu}: g \in G\right\}$ acts on $\mathbb{D}$, producing a quotient Riemann surface $M^{\mu} \simeq \mathbb{D} / G^{\mu}$ such that the quotient map $\pi^{\mu}: \mathbb{D} \rightarrow M^{\mu}$ is a holomorphic universal covering. By (2.4), $f^{\mu}$ projects to a quasiconformal map $\tilde{f}^{\mu}$ of $M$ onto $M^{\mu}$ such that $\tilde{f}^{\mu} \circ \pi=\pi^{\mu} \circ f^{\mu}$. By definition, $[\mu]_{T(M)}$ is the Teichmüller equivalence class of $\tilde{f}^{\mu}$. This procedure gives a map $\mu \mapsto[\mu]_{T(M)}$ of $B(\mathbb{D}, G)$ onto $T(M)$. Similarly, the map $\mu \mapsto[\mu]_{A T(M)}$ is defined by setting $[\mu]_{A T(M)}$ equal to the asymptotic equivalence class of $\widetilde{f}^{\mu}$.

The following proposition summarises some well-known properties of these maps. See, for example, 4 for the proof.

Proposition 2.1. The map $\mu \mapsto[\mu]_{T(M)}$ from $B(\mathbb{D}, G)$ to $T(M)$ is surjective and for $\mu, \nu \in B(\mathbb{D}, G),[\mu]_{T(M)}=[\nu]_{T(M)}$ if and only if $f^{\mu}=f^{\nu}$ on $\partial \mathbb{D}$.

The map $\mu \mapsto[\mu]_{A T(M)}$ from $B(\mathbb{D}, G)$ to $A T(M)$ is surjective and for $\mu, \nu \in$ $B(\mathbb{D}, G),[\mu]_{A T(M)}=[\nu]_{A T(M)}$ if and only if there exists $\widetilde{\mu}, \widetilde{\nu} \in B(\mathbb{D}, G)$ such that $[\widetilde{\mu}]_{T(M)}=[\mu]_{T(M)}$, $[\widetilde{\nu}]_{T(M)}=[\nu]_{T(M)}$ and $\widetilde{f}^{\widetilde{\mu}} \circ\left(\widetilde{f^{\nu}}\right)^{-1}: M^{\widetilde{\nu}} \rightarrow M^{\widetilde{\mu}}$ is asymptotically conformal.

For $\mu \in L^{\infty}(M)$ and $E \subseteq M$ measurable, the $L^{\infty}$ norm of $\left.\mu\right|_{E}$ will be denoted by $\|\mu\|_{E}$. Recalling the correspondence between $L^{\infty}(M)$ and $L^{\infty}(\mathbb{D}, G)$, we will say that $\mu \in L^{\infty}(M)$ vanishes at infinity on $M$ if and only if for all $\epsilon>0$, there exists a compact set $E \subset M$ such that $\|\mu\|_{M \backslash E}<\epsilon$. The set of $\mu$ that vanish at infinity on $M$ forms a closed subspace of $L^{\infty}(M)$, denoted by $L_{0}^{\infty}(M)$. The following result is proved in [3].

Lemma 2.2. For $\mu, \nu \in B(M),[\mu]_{A T(M)}=[\nu]_{A T(M)}$ if and only if there exists $\widetilde{\mu}, \widetilde{\nu} \in B(\mathbb{D}, G)$ such that $[\widetilde{\mu}]_{T(M)}=[\mu]_{T(M)},[\widetilde{\nu}]_{T(M)}=[\nu]_{T(M)}$ and $\widetilde{\mu}-\widetilde{\nu}$ vanishes at infinity on $M$.

This implies that the map $\mu \mapsto[\mu]_{A T(M)}$ from $B(M)$ to $A T(M)$ factors through the natural projection of $B(M)$ onto the open unit ball of $L^{\infty}(M) / L_{0}^{\infty}(M)$.

2.4. Schwarzian derivatives and the Bers embedding. For $\mu \in B(\mathbb{D}, G)$, let $f_{\mu}$ be the quasiconformal map of $\widehat{\mathbb{C}}$ onto itself that fixes $1,-1, i$, is conformal on $\mathbb{D}^{*}$ and has Beltrami differential $\mu$ in $\mathbb{D}$. Since $f_{\mu}$ is conformal in $\mathbb{D}^{*}$, we can form its Schwarzian derivative

$$
\varphi_{\mu}(z)=S\left(f_{\mu}\right)(z)=\left(\frac{f_{\mu}^{\prime \prime}(z)}{f_{\mu}^{\prime}(z)}\right)^{\prime}-\frac{1}{2}\left(\frac{f_{\mu}^{\prime \prime}(z)}{f_{\mu}^{\prime}(z)}\right)^{2},
$$

for $z \in \mathbb{D}^{*}$. It is well known that $\varphi_{\mu} \in Q\left(\mathbb{D}^{*}, G\right)$. Since $\varphi_{\mu}$ satisfies (2.2), they project to quadratic differentials on $M^{*} \simeq \mathbb{D}^{*} / G$, the conjugate surface to $M$, and so we can identify $Q\left(M^{*}\right)$ and $Q_{0}\left(M^{*}\right)$ with $Q\left(\mathbb{D}^{*}, G\right)$ and $Q_{0}\left(\mathbb{D}^{*}, G\right)$, respectively. 
The following proposition describes the Bers embedding. See the standard literature quoted in the introduction for the proof.

Proposition 2.3. For $\mu \in B(\mathbb{D}, G)$, the map $S_{M}(\mu)=\varphi_{\mu}$ defines a holomorphic map $S_{M}$ of $B(\mathbb{D}, G)$ onto a bounded open subset of $Q\left(\mathbb{D}^{*}, G\right)$. Further, $S_{M}(\mu)=$ $S_{M}(\nu)$ if and only if $[\mu]_{T(M)}=[\nu]_{T(M)}$ and it follows that $S_{M}$ induces a holomorphic map $\widetilde{S}_{M}$ from $T(M)$ onto a bounded open subset of $Q\left(\mathbb{D}^{*}, G\right)$, called the Bers embedding.

Recalling the little Bers space $Q_{0}\left(\mathbb{D}^{*}, G\right)$ defined in the introduction, write $\widehat{Q}(G)$ for the quotient Banach space $Q\left(\mathbb{D}^{*}, G\right) / Q_{0}\left(\mathbb{D}^{*}, G\right)$ and $P_{Q}$ for the quotient map from $Q\left(\mathbb{D}^{*}, G\right)$ to $\widehat{Q}(G)$. Note that we can identify $\widehat{Q}(G)$ with $\widehat{Q}\left(M^{*}\right)$ in the notation of $\S 2.1$.

Proposition 2.4. The formula $\chi_{M}\left([\mu]_{A T(M)}\right)=P_{Q}\left(S_{M}(\mu)\right)$ for $\mu \in B(\mathbb{D}, G)$ gives a well-defined injective map $\chi_{M}$ of $A T(M)$ onto a bounded open subset of $\widehat{Q}(G)$ and $A T(M)$ has a unique complex structure such that $\chi_{M}$ is biholomorphic.

The map $\chi_{M}$ is called the asymptotic Bers embedding. See [2, 3] for the locally injective and locally holomorphic properties of $\chi_{M}$ and [4] for the globally holomorphic property.

\section{Isomorphism Class of the little Bers space}

3.1. Bergman kernels. Recall that $\mathbb{D}$ is the unit disk and $\rho(z)$ is the hyperbolic density on $\mathbb{D}$. Let $\sigma=\sigma_{\Omega}$ denote area measure on the domain or Riemann surface $\Omega$. We will suppress the subscript where there is no confusion as to the region under consideration. More information on Bergman kernels can be found in, for example, [1, 7. The following lemma is standard and is proved for the reader's convenience.

Lemma 3.1. The Bergman kernel on $\mathbb{D} \times \mathbb{D}$ is given by

$$
K(z, \zeta)=\frac{1}{(1-z \bar{\zeta})^{4}}
$$

and satisfies the following properties:

(i) for all $z, \zeta \in \mathbb{D}$, we have $K(z, \zeta)=\overline{K(\zeta, z)}$;

(ii) for every Möbius transformation $A: \mathbb{D} \rightarrow \mathbb{D}$,

$$
K(A(z), A(\zeta)) A^{\prime}(z)^{2} \overline{A^{\prime}(\zeta)^{2}}=K(z, \zeta)
$$

(iii) for $z \in \mathbb{D}$,

$$
\int_{\mathbb{D}}|K(z, \zeta)| d \sigma(\zeta) \leq \frac{\pi}{4} \rho^{2}(z)
$$

(iv) if $f$ is analytic in $\mathbb{D}$ and satisfies

$$
\int_{\mathbb{D}}|f(\zeta)|\left(1-|\zeta|^{2}\right)^{2} d \sigma(\zeta)<\infty
$$

noting that this condition is satisfied if $f \in A^{1}(\mathbb{D})$ or $f \in Q(\mathbb{D})$, then we have

$$
f(z)=\frac{12}{\pi} \int_{\mathbb{D}} \rho^{-2}(\zeta) K(z, \zeta) f(\zeta) d \sigma(\zeta) ;
$$

(v) for each $\zeta \in \mathbb{D}$,

$$
\sup _{z \in \mathbb{D}}|K(z, \zeta)| \rho^{-2}(z)<\infty .
$$


Proof. The first property is obvious from the definition of $K$. The second property follows from an elementary calculation. For the third property, consider

$$
h(\zeta)=\int_{\mathbb{D}}|K(z, \zeta)| d \sigma(z)
$$

and observe using (ii) that under the change of variable $\zeta \mapsto A(\zeta)$, for a Möbius transformation $A: \mathbb{D} \rightarrow \mathbb{D}$, we have $h(A(\zeta))\left|A^{\prime}(\zeta)\right|^{2}=h(\zeta)$. Therefore $h(\zeta)$ can be determined by evaluating $h(0)$. Since $h(0)=\pi$, this gives the third property with equality. For the fourth property, note first that $\left(1-|w|^{2}\right)^{2} d \sigma(w)$ is a radial measure of norm $\pi / 3$ and so the mean value property implies that

$$
f(0)=\frac{3}{\pi} \int_{\mathbb{D}} f(w)\left(1-|w|^{2}\right)^{2} d \sigma(w) .
$$

Let $A_{z}(w)$ be the Möbius transformation $(z-w) /(1-\bar{z} w)$ which maps 0 to $z$ and apply (3.1) to $f \circ A_{z}$ to give

$$
\begin{aligned}
f(z) & =\frac{3}{\pi} \int_{\mathbb{D}} f\left(A_{z}(w)\right)\left(1-|w|^{2}\right)^{2} d \sigma(w) \\
& =\frac{3}{\pi} \int_{\mathbb{D}} f(\zeta)\left(1-\left|A_{z}(\zeta)\right|^{2}\right)^{4}\left(1-|\zeta|^{2}\right)^{-2} d \sigma(\zeta) \\
& =\frac{3}{\pi}\left(1-|z|^{2}\right)^{4} \int_{\mathbb{D}} f(\zeta)\left(1-|\zeta|^{2}\right)^{2}|1-\bar{z} \zeta|^{-8} d \sigma(\zeta),
\end{aligned}
$$

by using $w=A_{z}(\zeta)$ and the fact that

$$
1-\left|A_{z}(\zeta)\right|^{2}=\frac{\left(1-|z|^{2}\right)\left(1-|\zeta|^{2}\right)}{|1-\bar{z} \zeta|^{2}} .
$$

Applying (3.2) to the function $(1-\bar{z} t)^{4} f(t)$ then gives

$$
f(z)=\frac{3}{\pi} \int_{\mathbb{D}} f(\zeta)(1-\bar{\zeta} z)^{-4}\left(1-|\zeta|^{2}\right)^{2} d \sigma(\zeta)=\frac{12}{\pi} \int_{\mathbb{D}} f(\zeta) K(z, \zeta) \rho^{-2}(\zeta) d \sigma(\zeta) .
$$

The final property is obvious from the definitions of $K$ and $\rho$.

We define the operator $P$ by

$$
(P h)(z)=\int_{\mathbb{D}} K(z, \zeta) h(\zeta) d \sigma(\zeta)
$$

for $h \in L^{\infty}(\mathbb{D})$. Also let $\mathcal{T}$ be the operator on $L^{\infty}(\mathbb{D})$ given by

$$
(\mathcal{T} h)(z)=\rho^{-2}(z) h(z)
$$

for $h \in L^{\infty}(\mathbb{D})$.

Lemma 3.2. The operator $P$ maps $L^{\infty}(\mathbb{D})$ onto $Q(\mathbb{D})$ and $\mathcal{T} \circ P$ is a bounded operator. Further, $\left.P\right|_{C_{0}(\mathbb{D})}$ maps $C_{0}(\mathbb{D})$ onto $Q_{0}(\mathbb{D})$ and $\left.\mathcal{T} \circ P\right|_{C_{0}(\mathbb{D})}$ is a projection from $C_{0}(\mathbb{D})$ onto $\mathcal{T}\left(Q_{0}(\mathbb{D})\right)$.

Proof. We have

$$
\begin{aligned}
& |(P h)(z)|=\left|\int_{\mathbb{D}} K(z, \zeta) h(\zeta) d \sigma(\zeta)\right| \\
& \quad \leq\|h\|_{\infty} \int_{\mathbb{D}}|K(z, \zeta)| d \sigma(\zeta) \leq \frac{\pi|| h \|_{\infty}}{4} \rho^{2}(z)
\end{aligned}
$$


using properties (i) and (iii) of Lemma 3.1 This shows that $P h \in Q(\mathbb{D})$ and $\mathcal{T} \circ P$ is bounded with $\|\mathcal{T} \circ P\| \leq \pi / 4$. Now let $h \in C_{0}(\mathbb{D})$. Given $\epsilon>0$, choose $R \in(0,1)$ such that $|h(z)|<\epsilon$ for $|z|>R$. Then

$$
|(P h)(z)| \leq\left(\int_{|z| \leq R}+\int_{|z|>R}\right)|K(z, \zeta) h(\zeta)| d \sigma(\zeta)=I_{1}+I_{2} .
$$

From (3.3), $I_{2} \leq \epsilon \pi \rho^{2}(z) / 4$. Also, $I_{1} \leq a_{R}\|h\|_{\infty}$, where $a_{R}$ is a constant which depends only on $R$. Hence

$$
\rho^{-2}(z)|(P h)(z)| \leq a_{R}\|h\|_{\infty} \rho^{-2}(z)+\epsilon \pi / 4
$$

for $z \in \mathbb{D}$. Thus

$$
\limsup _{|z| \rightarrow 1} \rho^{-2}(z)|(P h)(z)| \leq \epsilon \pi / 4
$$

and so $P$ maps $C_{0}(\mathbb{D})$ into $Q_{0}(\mathbb{D})$. Finally, $P(\mathcal{T}(P h))=P h$ by property (iv) of Lemma 3.1 and therefore $\mathcal{T} \circ P$ is a projection of $C_{0}(\mathbb{D})$ onto $\mathcal{T}\left(Q_{0}(\mathbb{D})\right)$.

Every hyperbolic Riemann surface $M$ has the disk $\mathbb{D}$ as its universal cover. That is, there is a covering group $G$ such that $M \simeq \mathbb{D} / G$. Now, given such a covering group $G$ and a fundamental region $\Omega$ for $G$, form the Poincaré theta series given by

$$
F(z, \zeta)=\sum_{g \in G} K(g(z), \zeta) g^{\prime}(z)^{2} .
$$

The following lemma is standard and is proved for the reader's convenience.

Lemma 3.3. The series for $F(z, \zeta)$ converges absolutely and uniformly on compact subsets of $\mathbb{D}$ to a function holomorphic in $z$, antiholomorphic in $\zeta$, and satisfies

(i) $F(z, \zeta)=\overline{F(\zeta, z)}$;

(ii) for $g \in G, F(g(z), \zeta) g^{\prime}(z)^{2}=F(z, \zeta)$;

(iii) for $A$ in the normalizer of $G, F(A(z), A(\zeta)) A^{\prime}(z)^{2} \overline{A^{\prime}(\zeta)^{2}}=F(z, \zeta)$;

(iv) for every holomorphic quadratic differential $\psi$ which satisfies (2.2) and

$$
\int_{\Omega}|\psi(\zeta)|\left(1-|\zeta|^{2}\right)^{2}<\infty
$$

we have

$$
\psi(z)=\frac{12}{\pi} \int_{\Omega} \rho^{-2}(\zeta) F(z, \zeta) \psi(\zeta) d \sigma(\zeta) ;
$$

(v) for a fixed $|\zeta|<1$,

$$
\sup _{z \in \mathbb{D}}\left|F(z, \zeta) \rho^{-2}(z)\right|<\infty .
$$

Proof. For the fact that $F(z, \zeta)$ converges absolutely and uniformly on compact subsets, we refer to pages 78-82 of [7. Also, $F$ is clearly holomorphic in $z$ and antiholomorphic in $\zeta$. Property (i) follows from the symmetry property of $K$ and the fact that

$$
K(g(z), g(\zeta)) g^{\prime}(z)^{2} \overline{g^{\prime}(\zeta)^{2}}=K(z, \zeta)
$$


Property (ii) follows from the definition of $F$. To prove property (iii), let $A$ be in the normalizer of $G$. Then

$$
\begin{aligned}
F & (A(z), A(\zeta)) A^{\prime}(z)^{2} \overline{A^{\prime}(\zeta)^{2}}=\sum_{g \in G} K(g(A(z)), A(\zeta)) g^{\prime}(A(z))^{2} A^{\prime}(z)^{2} \overline{A^{\prime}(\zeta)^{2}} \\
& =\sum_{g \in G} K(g(A(z)), A(\zeta))(g \circ A)^{\prime}(z)^{2} \overline{A^{\prime}(\zeta)^{2}} \\
& =\sum_{g \in G} K\left(\left(A^{-1} \circ g \circ A\right)(z), \zeta\right)\left(A^{-1} \circ g \circ A\right)^{\prime}(z)^{2}=F(z, \zeta) .
\end{aligned}
$$

The invariance property (ii) of $F(z, \zeta)$ and the invariance

$$
(\rho \circ A)\left|A^{\prime}\right|=\rho
$$

mean that the formula in (iv) reduces to the case for $\mathbb{D}$, which was proved in Lemma 3.1. Finally, to prove (v), note that for a fixed $\zeta \in \mathbb{D}$,

$$
\sup _{z \in \mathbb{D}}|K(z, \zeta)|=\sup _{z \in \mathbb{D}} \frac{1}{|1-\bar{\zeta} z|^{4}}=\frac{1}{(1-|\zeta|)^{4}} .
$$

Therefore,

$$
\sup _{z \in \mathbb{D}}\left|F(z, \zeta) \rho^{-2}(z)\right| \leq \sup _{z \in \mathbb{D}} \rho^{-2}(z) \sum_{\gamma \in G}\left|\gamma^{\prime}(z)^{2}\right|(1-|\zeta|)^{-4},
$$

and so it is enough to show that

$$
\sum_{\gamma \in G}\left|\gamma^{\prime}(z)^{2}\right| \leq C \rho^{2}(z)
$$

for some constant $C$ depending only on the group $G$. Let

$$
h(z)=\frac{1}{\pi} \int_{\Omega} \frac{d \sigma(\zeta)}{|1-z \bar{\zeta}|^{4}},
$$

recalling $\Omega$ is a fundamental domain for $G$. By putting $f(\zeta)=(1-\zeta \bar{z})^{-2}$ in the reproduction formula given in property (iii) of Lemma 3.1,

$$
\sum_{\gamma \in G} h(\gamma(z))\left|\gamma^{\prime}(z)\right|^{2}=\frac{1}{\pi} \int_{\mathbb{D}} \frac{d \sigma(\zeta)}{|1-z \bar{\zeta}|^{4}}=\frac{1}{\left(1-|z|^{2}\right)^{2}} .
$$

The denominator of the integrand is less than $2^{4}=16$ and so

$$
h(z) \geq \frac{1}{16 \pi}|\Omega|,
$$

where $|\Omega|$ is the Euclidean area of $\omega$. This shows that (3.4) is true with

$$
C=\frac{16 \pi}{|\Omega|} \text {. }
$$

Let $M$ be a hyperbolic Riemann surface with covering group $G$ of $\mathbb{D}$ over $M$ and let $\pi: \mathbb{D} \rightarrow M$ be the projection from the universal cover to $M$. The Bergman kernel function $K_{M}$ for $M \times M$ is given by the projection of $F$ to $M \times M$. Since for any $g \in G$, we have $F(g(z), g(\zeta)) g^{\prime}(z)^{2} \overline{g^{\prime}(\zeta)^{2}}=F(z, \zeta)$, this projection is well defined. Recall $\rho_{M}$ is the hyperbolic density on $M$. 
Lemma 3.4. The kernel function $K_{M}: M \times M \rightarrow \mathbb{C}$ is holomorphic in the first argument, antiholomorphic in the second argument, and satisfies the following properties, where $p, q \in M$ :

(i) $K_{M}(p, q)=\overline{K_{M}(q, p)}$;

(ii) for every conformal $f: M \rightarrow M, K_{M}(f(p), f(q)) f^{\prime}(p)^{2} \overline{f^{\prime}(q)^{2}}=K_{M}(p, q)$;

(iii) $\int_{M}\left|K_{M}(p, q)\right| d \sigma(q) \leq \frac{\pi}{4} \rho_{M}^{2}(p)$;

(iv) for every holomorphic quadratic differential $\varphi$ on $M$ which satisfies

$$
\int_{M}|\varphi(q)| \rho_{M}^{-2}(q)<\infty
$$

including in particular the cases $\varphi \in A^{1}(M)$ and $\varphi \in Q(M)$, we have

$$
\varphi(p)=\frac{12}{\pi} \int_{M} \rho_{M}^{-2}(q) K_{M}(p, q) \varphi(q) d \sigma(q) ;
$$

(v) for each fixed $q \in M$,

$$
\sup _{p \in M}\left|K_{M}(p, q)\right| \rho_{M}^{-2}(p)<\infty .
$$

Proof. Most of the properties follow from the analogous properties of $F$, and here we will just prove the third property. Let $p=\pi(z)$ and $q=\pi(\zeta)$ for $p, q \in M$ and $z, \zeta \in \Omega$. We have

$$
\begin{aligned}
& \int_{M}\left|K_{M}(p, q)\right| d \sigma(p)=\int_{\Omega}|F(z, \zeta)| d \sigma(z) \\
& =\int_{\Omega}\left|\sum_{\gamma \in G} K_{\mathbb{D}}(\gamma(z), \zeta) \gamma^{\prime}(z)^{2}\right| d \sigma(z) \leq \sum_{\gamma \in G} \int_{\gamma(\Omega)}\left|K_{\mathbb{D}}(z, \zeta)\right| d \sigma(z) \\
& \quad=\int_{\mathbb{D}}\left|K_{\mathbb{D}}(z, \zeta)\right| d \sigma(z) \leq \frac{\pi}{4} \rho(z)^{2}=\frac{\pi}{4} \rho_{M}(p)^{2},
\end{aligned}
$$

where $\Omega$ is a fundamental region for $M$ in $\mathbb{D}$ and by using property (iii) of Lemma 3.1. which completes the proof.

Let $P_{M}: L^{\infty}(M) \rightarrow Q(M)$ be given by

$$
\left(P_{M} \widetilde{h}\right)(p)=\int_{M} K_{M}(p, q) \widetilde{h}(q) d \sigma(q)
$$

for $\widetilde{h} \in L^{\infty}(M)$. Also let $\mathcal{T}_{M}: Q(M) \rightarrow L^{\infty}(M)$ be the operator given by

$$
\left(\mathcal{T}_{M} \varphi\right)(p)=\rho_{M}^{-2}(p) \varphi(p)
$$

for $\varphi \in Q(M), p \in M$.

Lemma 3.5. The operator $P_{M}$ maps $L^{\infty}(M)$ onto $Q(M)$ and $\mathcal{T}_{M} \circ P_{M}$ is a bounded operator. Further, we have that $\left.P_{M}\right|_{C_{0}(M)}$ maps $C_{0}(M)$ onto $Q_{0}(M)$ and that $\left.\mathcal{T}_{M} \circ P_{M}\right|_{C_{0}(M)}$ is a projection from $C_{0}(M)$ onto $\mathcal{T}_{M}\left(Q_{0}(M)\right)$.

Proof. Recalling $M \simeq \mathbb{D} / G$ and $\pi: \mathbb{D} \rightarrow M$, let $\Omega$ be a fundamental region for $M$ in $\mathbb{D}$ containing 0 . Letting $p \in M$ and $p=\pi(z)$ for $z \in \Omega$, then

$$
\left|\left(P_{M} \widetilde{h}\right)(p)\right|=\left|\int_{M} K_{M}(p, q) \widetilde{h}(q) d \sigma(q)\right| \leq \frac{\pi \mid \widetilde{h} \|_{\infty}}{4} \rho_{M}^{2}(p)
$$

by property (iii) of Lemma 3.4. This shows that $P_{M}: L^{\infty}(M) \rightarrow Q(M)$ and that $\mathcal{T}_{M} \circ P_{M}$ is bounded with $\left\|\mathcal{T}_{M} \circ P_{M}\right\| \leq \pi / 4$. 
Now let $\widetilde{h} \in C_{0}(M)$ and let $h=\widetilde{h} \circ \pi$ be defined on $\Omega$. Clearly $\|\widetilde{h}\|_{\infty}=\|h\|_{\infty}$. Given $\epsilon>0$, choose $R \in(0,1)$ so that $|h(z)|<\epsilon$ for $z \in \Omega_{R}=\Omega \cap\{|z|>R\}$. Then

$$
\left|\left(P_{M} \widetilde{h}\right)(p)\right|=\left(\int_{\pi\left(\Omega \backslash \Omega_{R}\right)}+\int_{\pi\left(\Omega_{R}\right)}\right)\left|K_{M}(p, q) \widetilde{h}(q)\right| d \sigma(q) .
$$

Firstly,

$$
\begin{aligned}
& \int_{\pi\left(\Omega \backslash \Omega_{R}\right)}\left|K_{M}(p, q) \widetilde{h}(q)\right| d \sigma(q) \leq \int_{\Omega \backslash \Omega_{R}}|F(z, \zeta) h(\zeta)| d \sigma(\zeta) \\
& \quad \leq\|\widetilde{h}\|_{\infty} \sum_{g \in G} \int_{\Omega \backslash \Omega_{R}}\left|K(z, \zeta) \| g^{\prime}(\zeta)^{2}\right| d \sigma(\zeta) \\
& \quad \leq\left(\sup _{\zeta \in \Omega \backslash \Omega_{R}} \sum_{g \in G}\left|g^{\prime}(\zeta)\right|^{2}\right)\|\widetilde{h}\|_{\infty} \int_{\Omega \backslash \Omega_{R}}|K(z, \zeta)| d \sigma(\zeta) \\
& \quad \leq \frac{16 \pi C_{1}\|\widetilde{h}\|_{\infty} \rho^{2}(R)}{\left|\Omega \backslash \Omega_{R}\right|}
\end{aligned}
$$

using (3.4), (3.5) and the fact that $\int_{\Omega \backslash \Omega_{R}}|K(z, \zeta)| d \sigma(\zeta)$ is bounded by some constant $C_{1}$ depending only on $G$ and $R$. Now,

$$
\begin{aligned}
& \int_{\pi\left(\Omega_{R}\right)}\left|K_{M}(p, q) \widetilde{h}(q)\right| d \sigma(q) \leq \epsilon \int_{\Omega_{R}}|F(z, \zeta)| d \sigma(\zeta) \\
& \quad \leq \epsilon \sum_{g \in G} \int_{g\left(\Omega_{R}\right)}|K(z, \zeta)| d \sigma(\zeta) \\
& \quad \leq \epsilon \int_{|z|>R}|K(z, \zeta)| d \sigma(\zeta) \leq \frac{\pi \epsilon \rho^{2}(z)}{4}=\frac{\pi \epsilon \rho_{M}^{2}(p)}{4}
\end{aligned}
$$

by using property (iii) of Lemma 3.1. Therefore

$$
\rho_{M}^{-2}(p)\left|\left(P_{M} \widetilde{h}\right)(p)\right| \leq \frac{16 \pi C_{1}|| \widetilde{h} \|_{\infty} \rho^{2}(R)}{\left|\Omega \backslash \Omega_{R}\right|}+\frac{\pi \epsilon \rho_{M}^{2}(p)}{4} .
$$

Therefore

$$
\limsup _{\pi^{-1}(p) \in \Omega,\left|\pi^{-1}(p)\right| \rightarrow 1}\left|\left(P_{M} \widetilde{h}\right)(p)\right| \rho_{M}^{-2}(p) \leq \frac{\pi \epsilon}{4},
$$

which shows that $P_{M} \widetilde{h} \in Q_{0}(M)$. Finally, property (iv) of Lemma 3.4 implies that

$$
P_{M}\left(\mathcal{T}_{M}\left(P_{M}(\widetilde{h})\right)\right)=\widetilde{h}
$$

and so $\mathcal{T}_{M} \circ P_{M}$ is a projection.

\subsection{Some lemmata on projections.}

Lemma 3.6. Let $\Omega$ be a simply connected relatively compact subset of a hyperbolic Riemann surface $M$. Given $\epsilon>0$, there exists an operator

$$
\psi: X=\left.C_{0}(M)\right|_{\Omega} \rightarrow L^{\infty}(\Omega)
$$

such that (i) $\|\psi\| \leq 1$, (ii) we have

$$
\left\|\psi\left(\mathcal{T}_{M}\left(\left.\varphi\right|_{\Omega}\right)\right)-\mathcal{T}_{M}\left(\left.\varphi\right|_{\Omega}\right)\right\|<\epsilon
$$


for all $\varphi \in Q_{0}(M)$ satisfying $\|\varphi\|_{Q} \leq 1$, and (iii) $\psi\left(\mathcal{T}_{M}\left(\left.Q_{0}(M)\right|_{\Omega}\right)\right)$ is isometric to $\left(l^{\infty}\right)_{n}$, where $\left(l^{\infty}\right)_{n}$ is the $n$-dimensional subspace of $l^{\infty}$ with all terms, except possibly the first $n$ being equal to 0.

Proof. We can lift $M$ to the unit disk, so without loss of generality, we can assume that $\Omega$ is a relatively compact, simply connected subdomain of $\mathbb{D}$. Subdivide $\Omega$ into a finite number of disjoint simply connected subsets $\Omega_{1}, \ldots, \Omega_{n}$ and for $j=1, \ldots, n$ pick a point $w_{j} \in \Omega_{j}$. Define

$$
\psi\left(\left.f\right|_{\Omega}\right)=\sum_{j=1}^{n} f\left(w_{j}\right) \mathbf{1}_{\Omega_{j}},
$$

for $f \in C_{0}(\mathbb{D})$, where $\mathbf{1}_{\Omega_{j}}$ denotes the indicator function of $\Omega_{j}$. The operator $\psi$ is clearly linear, maps $X$ into $L^{\infty}(\Omega)$, is bounded and satisfies $\psi^{2}=\psi$. Therefore $\psi$ is a projection. We can define a map $\mu: \psi(X) \rightarrow\left(l^{\infty}\right)_{n}$ given by

$$
\mu(\psi(f))=\left(f\left(w_{1}\right), \ldots, f\left(w_{n}\right), 0, \ldots\right) .
$$

Now,

$$
\|\mu(\psi(f))\|_{l^{\infty}}=\max _{j=1, \ldots, n}\left|f\left(w_{j}\right)\right| .
$$

Also,

$$
\|\psi(f)\|_{\infty}=\sup _{z \in \Omega}|(\psi f)(z)|=\max _{j=1, \ldots, n} \sup _{z \in \Omega_{j}}|\psi(f(z))|=\max _{j=1, \ldots, n}\left|f\left(w_{j}\right)\right| .
$$

Hence $\mu$ is isometric. Further, for any $\left(\lambda_{1}, \ldots, \lambda_{n}, 0, \ldots\right) \in\left(l^{\infty}\right)_{n}$ we can find $f \in X$ such that $f\left(w_{j}\right)=\lambda_{j}$ for $j=1, \ldots, n$ and so $P(X)$ is isometric to $\left(l^{\infty}\right)_{n}$. We now have to show that given $\epsilon>0$, we can find a fine enough subdivision of $\Omega$ so that for the corresponding projection $\psi,\|(\psi f)-f\|<\epsilon$ for $f=\rho^{-2} \varphi$ with $\varphi \in Q_{0}(\mathbb{D})$ and $\|\varphi\|_{Q} \leq 1$.

Let $B$ be the unit ball in $Q_{0}(\mathbb{D})$, that is,

$$
B=\left\{\varphi \in Q_{0}(\mathbb{D}):\|\varphi\|_{Q} \leq 1\right\} .
$$

Since $\Omega$ is relatively compact in $\mathbb{D}$, there exists a constant $C$ depending only on $\Omega$ such that

$$
\sup _{z \in \Omega} \rho^{2}(z) \leq C<\infty .
$$

Therefore, we have

$$
\sup _{\varphi \in B, z \in \Omega}|\varphi(z)| \leq C,
$$

which implies that $\left.Q_{0}(\mathbb{D})\right|_{\Omega}$ is a normal family by Montel's Theorem. The ArzelàAscoli Theorem then implies that $\left.Q_{0}(\mathbb{D})\right|_{\Omega}$ is equicontinuous. Since $\rho^{-2}$ is bounded on $\mathbb{D}$, it follows that $\left\{\left.\rho^{-2} \varphi\right|_{\Omega}: \varphi \in Q_{0}(\mathbb{D})\right\}$ is equicontinuous. That is, given $z \in \Omega$, for all $\tilde{\epsilon}>0$ and $\varphi \in Q_{0}(\mathbb{D})$, there exists $\delta>0$ such that if $\zeta \in \Omega$ and $|z-\zeta|<\delta$, then $\left|\rho^{-2}(z) \varphi(z)-\rho^{-2}(\zeta) \varphi(\zeta)\right|<\widetilde{\epsilon}$

Now given $f=\left.\rho^{-2} \varphi\right|_{\Omega}$ with $\varphi \in B$ and $\epsilon>0$, pick $\delta>0$ satisfying the following. Partition $\Omega$ into a finite number of simply connected subsets $\Omega_{1}, \ldots, \Omega_{n}$ with points $w_{j} \in \Omega_{j}$ for $j=1, \ldots, n$ such that the diameter of $\Omega_{j}$ is less than $\delta / 2$ for $j=1, \ldots, n$. 
Then

$$
\begin{aligned}
& \|\psi(f)-f\|_{\infty}=\sup _{z \in \Omega}|((\psi f)-f)(z)| \\
& \quad=\max _{j=1, \ldots, n} \sup _{z \in \Omega_{j}}\left|\left(f\left(w_{j}\right) \mathbf{1}_{\Omega_{j}}-f\right)(z)\right|<\epsilon
\end{aligned}
$$

by the fact that the diameters of the $\Omega_{j}$ are less than $\delta / 2$ and so for $z \in \Omega_{j}$, $\left|w_{j}-z\right|<\delta / 2$ and also by equicontinuity. This proves the lemma.

A subspace $Y$ of a Banach space $Z$ is called complemented if there exists a projection from $Z$ onto $Y$. For the proof of the following lemma, see for example Proposition 2.5 in 5 .

Lemma 3.7. Let $Y$ be a complemented subspace of a Banach space $Z$, and let $T: Y \rightarrow Z$ be a linear operator satisfying

$$
\left\|T-\left.I\right|_{Y}\right\|<\epsilon .
$$

Then if $\epsilon$ is sufficiently small, $T(Y)$ is closed and complemented in $Z$.

\subsection{Proof of Theorem 1.1.}

Proof. We first subdivide $M$ in an appropriate way. For every $p \in M$, there exists an open subset $U_{p} \subset M$ containing $p$, and a chart $\pi_{p}$ such that $\pi_{p}\left(U_{p}\right)$ is an open disk in $\mathbb{C}$ and $\pi_{p}(p)=0$. Let $V_{p}$ be an open simply connected set in $M$ whose closure is contained in $U_{p}$, so that in particular $\pi_{p}\left(V_{p}\right)$ is a relatively compact subset of $\pi_{p}\left(U_{p}\right)$.

As $p$ varies through $M,\left(V_{p}\right)_{p \in M}$ forms an open cover of $M$, and it is possible to find a countable subset $p_{1}, p_{2}, \ldots$ such that

$$
M=\bigcup_{i=1}^{\infty} V_{p_{i}} .
$$

Now modify the subsets $V_{p_{i}}$ to give a disjoint partition of $M$ in the following way: define $M_{1}=V_{p_{1}}$, and then inductively,

$$
M_{n}=V_{p_{n}} \backslash\left(\bigcup_{i=1}^{n-1} V_{p_{i}}\right) .
$$

Let $R_{i}: L^{\infty}(M) \rightarrow L^{\infty}\left(M_{i}\right)$ be the restriction map given by $R_{i}(f)=\left.f\right|_{M_{i}}$, for $f \in L^{\infty}(M)$. Each $M_{i}$ is a relatively compact subset of $M$ and so $R_{i}(f) \in L^{\infty}\left(M_{i}\right)$. Define the operator $R: L^{\infty}(M) \rightarrow\left(L^{\infty}\left(M_{1}\right) \oplus L^{\infty}\left(M_{2}\right) \oplus \ldots\right)_{\infty}$ by

$$
R(f)=\left(R_{1}(f), R_{2}(f), \ldots\right),
$$

for $f \in L^{\infty}(M)$. The operator $R$ is isometric, since

$$
\|R(f)\|:=\sup _{i \in \mathbb{N}}|| R_{i}(f)\left\|=\sup _{i \in \mathbb{N}, p \in M_{i}}|f(p)|=\sup _{p \in M}|f(p)|=\right\| f \|_{\infty},
$$

and $R$ is also clearly surjective. We now consider $R$ restricted to $C_{0}(M)$.

By Lemma 3.6 given $\epsilon_{i}>0$ we can find an operator

$$
\psi_{i}: R_{i}\left(C_{0}(M)\right) \rightarrow L^{\infty}\left(M_{i}\right)
$$

such that $\left\|\psi_{i}\right\| \leq 1$ and

$$
\left\|\psi_{i}\left(R_{i}\left(\rho_{M}^{-2} \varphi\right)\right)-R_{i}\left(\rho_{M}^{-2} \varphi\right)\right\| \leq \epsilon_{i}
$$


for all $\varphi \in Q_{0}(M)$ with $\|\varphi\|_{Q} \leq 1$. Further, if $f \in C_{0}(M)$, then $\left\|R_{i}(f)\right\|_{\infty} \rightarrow 0$ as $i \rightarrow \infty$ by the construction of the $M_{i}$. Also $\left\|\psi_{i}\left(R_{i}(f)\right)\right\|_{\infty} \rightarrow 0$ as $i \rightarrow \infty$ because $\left\|\psi_{i}\right\| \leq 1$. Using this and since $\psi_{i}\left(R_{i}\left(\mathcal{T}_{M}\left(C_{0}(M)\right)\right)\right)$ is isometric to $\left(l^{\infty}\right)_{n_{i}}$ for some $n_{i} \in \mathbb{N}$ by Lemma 3.6, if we let

$$
\Lambda=\left(\psi_{1}\left(R_{1}\left(C_{0}(M)\right)\right) \oplus \psi_{2}\left(R_{2}\left(C_{0}(M)\right)\right) \oplus \ldots\right)_{\infty},
$$

it follows that $\Lambda$ is isometric to $c_{0}$. Now define the operator $\alpha: R\left(\mathcal{T}_{M}\left(Q_{0}(M)\right)\right) \rightarrow \Lambda$ by

$$
\alpha\left(R_{1}(f), R_{2}(f), \ldots\right)=\left(\psi_{1}\left(R_{1}(f)\right), \psi_{2}\left(R_{2}(f)\right), \ldots\right),
$$

for $f=\rho_{M}^{-2} \varphi$, where $\varphi \in Q_{0}(M)$. Since the dimension of $Q_{0}(M)$ is infinite, $R\left(\mathcal{T}_{M}\left(Q_{0}(M)\right)\right)$ also must be infinite dimensional. We also have

$$
\|\alpha(\xi)-\xi\| \leq\left(\sum_{i=1}^{\infty} \epsilon_{i}\right)\|\xi\|
$$

for $\xi \in R\left(\mathcal{T}_{M}\left(Q_{0}(M)\right)\right)$, and so given $\epsilon>0$, it is possible to choose the $\left(\epsilon_{i}\right)_{i=1}^{\infty}$ so that $\|\alpha(\xi)-\xi\|<\epsilon\|\xi\|$, for $\xi \in R\left(\mathcal{T}_{M}\left(Q_{0}(M)\right)\right)$.

By Lemma 3.5. $\mathcal{T}_{M} \circ P_{M}: C_{0}(M) \rightarrow \mathcal{T}_{M}\left(Q_{0}(M)\right)$ is a bounded linear projection Therefore, there is a bounded linear projection $\theta: R\left(C_{0}(M)\right) \rightarrow R\left(\mathcal{T}_{M}\left(Q_{0}(M)\right)\right)$, given by

$$
\theta\left(R_{1}(f), R_{2}(f), \ldots\right)=\left(R_{1}\left(\mathcal{T}_{M} \circ P_{M}(f)\right), R_{2}\left(\mathcal{T}_{M} \circ P_{M}(f)\right), \ldots\right)
$$

which is clearly linear, bounded and satisfies $\theta^{2}=\theta$. Therefore $R\left(\mathcal{T}_{M}\left(Q_{0}(M)\right)\right)$ is complemented in $R\left(C_{0}(M)\right)$. Thus, by Lemma 3.7, if $\epsilon$ is chosen to be small enough, $\alpha\left(R\left(\mathcal{T}_{M}\left(Q_{0}(M)\right)\right)\right)$ is complemented in $R\left(C_{0}(M)\right)$ and, in particular, $\Lambda$. This follows since if $W \subset Y$ is complemented in $X$, then there exists a projection $S: X \rightarrow W,(\operatorname{Im}(S)) \cap Y$ is complemented in $Y$ and so $W$ is complemented in $Y$.

If $\epsilon<1$, then $\|\alpha-I\|<1$, and Lemma 3.7 gives that $\alpha$ is thus invertible and an isomorphism. By a classical result due to Pelczynski 21, every infinite dimensional complemented subspace of $c_{0}$ is isomorphic to $c_{0}$, and so $\mathcal{T}_{M}\left(Q_{0}(M)\right)$ is isomorphic to $c_{0}$. Clearly $\mathcal{T}_{M}: Q_{0}(M) \rightarrow \mathcal{T}_{M}\left(Q_{0}(M)\right) \subseteq L^{\infty}(M)$ is an isometry and so $Q_{0}(M)$ is isomorphic to $c_{0}$.

Finally, if $M$ and $N$ are Riemann surfaces of infinite analytic type, then $Q_{0}(M)$ and $Q_{0}(N)$ are both isomorphic to $c_{0}$ and hence are isomorphic to each other.

\section{Proof of Corollaries}

4.1. Proof of Corollary 1.2. It is well known that the Banach dual of $Q_{0}(M)$ can be identified with $A^{1}(M)$. Theorem 1.1 then implies results proved in [5], namely that $A^{1}(M)$ is isomorphic to $l^{1}$ and $Q(M)$ is isomorphic to $l^{\infty}$ if $M$ is a Riemann surface of infinite analytic type. Recalling that $\widehat{Q}(M)=Q(M) / Q_{0}(M)$, we immediately have an isomorphism $\widehat{\beta_{M}}: \widehat{Q}(M) \rightarrow l^{\infty} / c_{0}$.

If $N$ is also a Riemann surface of infinite analytic type, then ${\widehat{\beta_{N}}}^{-1} \circ{\widehat{\beta_{M}}}$ gives an isomorphism between $\widehat{Q}(M)$ and $\widehat{Q}(N)$.

4.2. Proof of Corollary 1.3. By Proposition 2.4, $\chi_{M}$ maps $A T(M)$ biholomorphically onto a bounded open subset of $\widehat{Q}\left(M^{*}\right)$. Therefore $\widehat{\beta_{M^{*}} \circ \chi_{M}}$ is a bi-Lipschitz mapping of $A T(M)$ onto a bounded open subset of $l^{\infty} / c_{0}$, since it is a composition of a biholomorphic mapping with an isomorphism. 
4.3. Proof of Theorem 1.4. Let $M$ and $N$ be two Riemann surfaces of infinite analytic type. Then there exist neighbourhoods $U_{M}, U_{N}$ of the base-points in $A T(M)$ and $A T(N)$ respectively such that $\widehat{\beta_{M^{*}}} \circ \chi_{M}\left(U_{M}\right)$ and $\widehat{\beta_{N^{*}}} \circ \chi_{N}\left(U_{N}\right)$ are neighbourhoods of the origin in $l^{\infty} / c_{0}$. Letting

$$
V=\left(\widehat{\beta_{M^{*}}} \circ \chi_{M}\left(U_{M}\right)\right) \cap\left(\widehat{\beta_{N^{*}}} \circ \chi_{N}\left(U_{N}\right)\right) \subset l^{\infty} / c_{0},
$$

it follows that

$$
\chi_{N}^{-1} \circ{\widehat{\beta_{N^{*}}}}^{-1} \circ \widehat{\beta_{M^{*}}} \circ \chi_{M}
$$

maps a neighbourhood $\chi_{M}^{-1} \circ{\widetilde{\beta_{M^{*}}}}^{-1}(V)$ of the origin of $A T(M)$ isomorphically onto a neighbourhood $\chi_{N}^{-1} \circ{\widehat{\beta_{N^{*}}}}^{-1}(V)$ of the origin of $A T(N)$. Noting that $\chi_{M}, \chi_{N}$ are biholomorphic and $\widehat{\beta_{M^{*}}}, \widehat{\beta_{M^{*}}}$ are isomorphisms, it follows that a neighbourhood of the base-point of $A T(M)$ is bi-Lipschitz equivalent to a neighbourhood of the base-point of $A T(N)$.

Recalling the notation following (2.4), in the complex Banach manifold structure of Teichmüller space, a chart for the neighbourhood of the base-point of $T\left(M^{\mu}\right)$ is a chart for the neighbourhood of $[\mu]_{T(M)}$ in $T(M)$ (see the standard references cited in the introduction). This implies that a chart for the neighbourhood of the base-point of $A T\left(M^{\mu}\right)$ is a chart for the neighbourhood of $[\mu]_{A T(M)}$ in $A T(M)$.

If $[\mu]_{A T(M)} \in A T(M)$ and $[\nu]_{A T(N)} \in A T(N)$, then we have a bi-Lipschitz equivalence between neighbourhoods of the base-points of $A T\left(M^{\mu}\right)$ and $A T\left(N^{\nu}\right)$ respectively by using the argument above. This then implies we have a bi-Lipschitz equivalence between neighbourhoods of $[\mu]_{A T(M)}$ and $[\nu]_{A T(N)} \in A T(N)$, which proves the theorem.

4.4. Proof of Corollary 1.5. Recall that the set of asymptotically conformal classes in $T(M)$ is denoted by $T_{0}(M)$. Recalling the notation of $\S 2.4, P_{Q} \circ \widetilde{S}_{M}$ and $\chi_{\chi_{M}} \circ \mathcal{P}$ are equal as mappings from $T(M)$ to $\widehat{Q}\left(M^{*}\right)$. Since the Bers embedding $\widetilde{S}_{M}$ and the asymptotic Bers embedding $\chi_{M}$ are biholomorphic, the fibres of $\mathcal{P}$ in $T(M)$ are homeomorphic to fibres of $P_{Q}$ in $\widetilde{S}_{M}(T(M)) \subset Q\left(M^{*}\right)$. Therefore $T_{0}(M)=\mathcal{P}^{-1}\left([0]_{A T(M)}\right)$ is homeomorphic to a subset of $P_{Q}^{-1}\left([0]_{l \infty} / c_{0}\right)=Q_{0}\left(M^{*}\right)$, which in turn is isomorphic to $c_{0}$ by Theorem 1.1. This completes the proof.

\section{REFERENCES}

[1] P.L.Duren and A.Schuster, Bergman Spaces, AMS Mathematical Surveys and Monographs, vol. 100, 2004. MR 2033762 (2005c:30053)

[2] C.J.Earle, F.P.Gardiner and N.Lakic, Teichmüller spaces with asymptotic conformal equivalence, I.H.E.S. preprint, 1995.

[3] C.J.Earle, F.P.Gardiner and N.Lakic, Asymptotic Teichmüller space, Part I: The complex structure, In the tradition of Ahlfors and Bers (Stony Brook, NY, 1998), 17-38, Contemp. Math. 256, Amer. Math. Soc., Providence, RI, 2000. MR.1759668 (2001m:32029)

[4] C.J.Earle, V.Markovic and D.Saric, Barycentric extension and the Bers embedding for asymptotic Teichmüller space, Complex manifolds and hyperbolic geometry (Guanajuato, 2001), 87-105, Contemp. Math., 311, Amer. Math. Soc., Providence, RI, 2002. MR 1940165 (2003i:30072)

[5] A.Fletcher, Local rigidity of infinite dimensional Teichmüller spaces, J. London Math. Soc. (2) 74, 26-40, 2006. MR2254550 (2007g:30066)

[6] A.Fletcher and V.Markovic, Quasiconformal maps and Teichmüller theory, Oxford Graduate Texts in Mathematics, 11, Oxford University Press, 2007. MR 2269887(2007g:30001)

[7] F.P.Gardiner, Teichmüller theory and quadratic differentials, John Wiley and Sons, Inc., New York, 1987. MR.903027 (88m:32044) 
[8] F.P.Gardiner and N.Lakic, Quasiconformal Teichmüller Theory, Math. Surveys Monogr., 76, Amer. Math. Soc., Providence, RI, 2000. MR1730906 (2001d:32016)

[9] F.P.Gardiner and D.P.Sullivan, Symmetric structures on a closed curve, Amer. J. Math., 114, 683-736, 1992. MR.1175689 (95h:30020)

[10] J.H.Hubbard, Teichmüller Theory and Applications to Geometry, Topology and Dynamics, Volume 1: Teichmüller Theory, Matrix Editions, NY, 2006. MR2245223 (2008k:30055)

[11] I.Kra, Automorphic forms and Kleinian groups, W. A. Benjamin, Reading, Mass., 1972. MR0357775 (50:10242)

[12] O.Lehto, Univalent functions and Teichmüller spaces, Graduate Texts in Mathematics, 109, Springer, New York, 1987. MR867407 (88f:30073)

[13] J.Lindenstrauss and A.Pelczynski, Contributions to the theory of the classical Banach space, J. Functional Analysis, 8, 225-249, 1971. MR0291772 (45:863)

[14] W.Lusky, On the structure of $H v_{0}(D)$ and $h v_{0}(D)$, Math. Nachr., 159, 279-289, 1992. MR:1237115 (94i:46040)

[15] W.Lusky, On the isomorphism classes of weighted spaces of harmonic and holomorphic functions, Studia Math., 175, no. 1, 19-45, 2006. MR2261698 (2007f:30089)

[16] V.Markovic, Biholomorphic maps between Teichmüller spaces, Duke Math. J., 120, no.2, 405-431, 2003. MR2019982(2004h:30058)

[17] M.Mateljević, The dual of the Bergman space defined on a hyperbolic plane domain, Publications de l'Institut Mathématique, 56(70), 135-139, 1994. MR1349080 (96e:46035)

[18] H.Miyachi, A reduction for asymptotic Teichmüller spaces, Ann. Acad. Sci. Fenn. Math., 32, 55-71, 2007. MR2297877 (2008j:32016)

[19] H.Miyachi, Image of Asymptotic Bers Map, J. Math. Soc. Japan, 60, No. 4, 1255-1276, 2008.

[20] S.Nag, The Complex Analytic Theory of Teichmüller Spaces, John Wiley and Sons, New York, 1988. MR.927291 (89f:32040)

[21] A.Pelczynski, Projections in certain Banach spaces, Studia Math., 19, 209-228, 1960. MR0126145 (23:A3441)

[22] J.Shapiro, Mackey topologies, reproducing kernels and diagonal maps on the Hardy and Bergman spaces, Duke. Math. J., 43, 187-202, 1976. MR0500100(58:17806)

[23] A.L.Shields and D.L.Williams, Bounded projections, duality, and multipliers in spaces of analytic functions, Trans. Amer. Math. Soc., 162, 287-302, 1971. MR0283559(44:790)

[24] P.Wojtaszczyk, $H_{p}$-spaces, $p \leq 1$, and spline systems, Studia Math., 77, no.3, 289-320, 1984 . MR745285 (85f:46053)

[25] G.Yao, Harmonic maps and asymptotic Teichmüller space, Manuscripta Math., 122, no. 4, 375-389, 2007. MR2300050 (2008e:37047)

Mathematics Institute, University of Warwick, Coventry, CV4 7AL, United Kingdom

E-mail address: Alastair.Fletcher@warwick.ac.uk 